\title{
Coercivity of 3D nanoscale magnetic arrays from self-assembly template methods
}

\author{
A.A. Zhukov ${ }^{\mathrm{a}}$, M.A. Ghanem ${ }^{\mathrm{b}}$, A.V. Goncharov ${ }^{\mathrm{a}}$, P.A.J. de Groot ${ }^{\mathrm{a}, *}$, \\ I.S. El-Hallag ${ }^{\mathrm{b}, 1}$, P.N. Bartlett ${ }^{\mathrm{b}}$, R. Boardman ${ }^{\mathrm{c}}$, H. Fangohr ${ }^{\mathrm{c}}$ \\ ${ }^{a}$ Department of Physics and Astronomy, University of Southampton, SO17 1BJ, UK \\ ${ }^{\mathrm{b}}$ Department of Chemistry, University of Southampton, Southampton SO17 1BJ, UK \\ ${ }^{\mathrm{c}}$ School of Engineering Sciences, University of Southampton, Southampton, SO17 1BJ, UK
}

\begin{abstract}
We present results of electrochemical deposition of magnetic metals and alloys in the pores of templates prepared by self-assembly from colloidal suspensions of polystyrene latex spheres. This technique has enabled us to create highly ordered magnetic nano-structures with 3D architectures on length scales ranging from $50-1000 \mathrm{~nm}$. Varying the parameters in the preparation (sphere diameter, film thickness and composition) allows us to produce materials with predetermined magnetic parameters.
\end{abstract}

(C) 2003 Elsevier B.V. All rights reserved.

PACS: 75.75. + a; 75.60.-d

Keywords: Nanostructures; Self-assembly; Anti-dots; Coercive field

Efficient methods to fabricate magnetic patterned structures are required for the development of magnetic media with high recording density. Combining thin film growth techniques with sub-micron lithography is a standard solution for this task. However, for lateral dimensions below 100-200 $\mathrm{nm}$ relatively cheap and fast optical lithography techniques become inapplicable. The cost of ion or electron lithography is uneconomically high for industrial applications. Alternative routes are needed for creating highly ordered magnetic structures on length scales down to a few nanometers. In this paper we present results for magnetic nanostructured samples prepared by electrochemical deposition through different templates produced by self-assembly of polystyrene spheres.

\footnotetext{
*Corresponding author. Tel.: +44-23-80592110; fax: +4423-80593910.

E-mail address: pajdeg@phys.soton.ac.uk (P.A.J. de Groot).

${ }^{1}$ On leave from the Chemistry Department, Faculty of Science, Tanta University, Tanta, Egypt.
}

Well-ordered templates have been prepared using a very slow (3-5days) evaporation of a colloidal water suspension containing $0.5 \mathrm{wt} \%$ of polystyrene spheres. The details of the preparation method are described in Refs. [1-3]. Magnetic films with pore size $50-1000 \mathrm{~nm}$ have been prepared. Fig. 1 presents a scanning electron microscope (SEM) image of a $\mathrm{Fe}_{0.5} \mathrm{Ni}_{0.5}$ film deposited trough a template of spheres with a diameter of $0.5 \mu \mathrm{m}$. The crystal structure, composition and morphology of the films were characterised using SEM, energy dispersive X-ray spectroscopy and X-ray diffraction.

Magnetic measurements have been performed using several techniques: vibrating sample, SQUID, Hall probe (sensitive area $50 \mu \mathrm{m} \times 50 \mu \mathrm{m}$ ) [4] magnetometers and magneto-optical Kerr effect rig. Most measurements have been done at room temperature with the magnetic field applied parallel to the film plane.

We have studied the impact of nanostructuring on the magnetic properties of different magnetic films of cobalt, iron, nickel and soft magnetic alloy $\mathrm{Fe}_{0.5} \mathrm{Ni}_{0.5}$ (both plain and patterned films had polycrystalline structure without texture). The magnetization loops revealed significant changes resulting from the nanoporous 


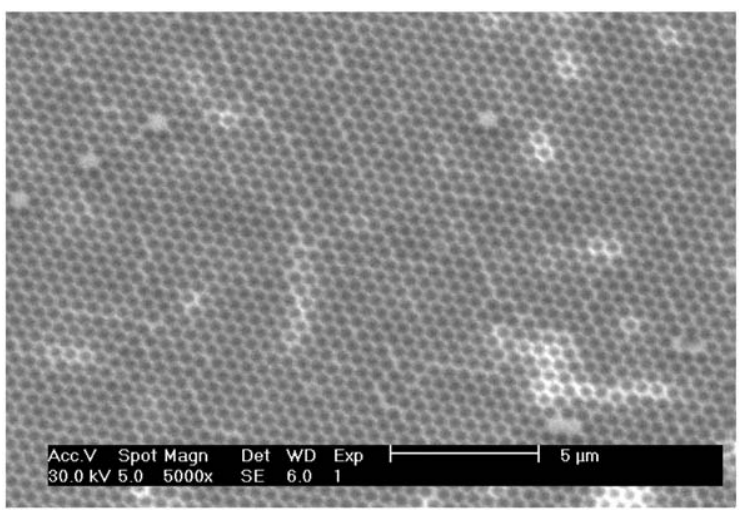

Fig. 1. SEM image of $\mathrm{Fe}_{0.5} \mathrm{Ni}_{0.5}$ film prepared using a template from $500 \mathrm{~nm}$ polystyrene spheres.
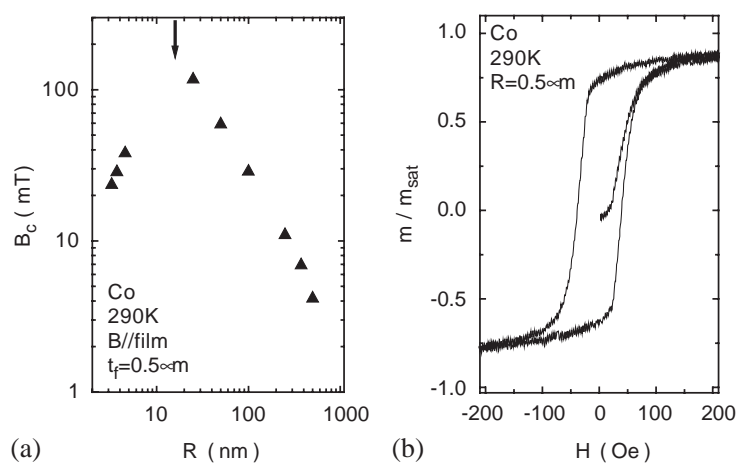

Fig. 2. (a) Dependence of coercive field in Co structured films on the radius of spherical pore (the arrow shows domain wall width, films with small pores $R<10 \mathrm{~nm}$ have been prepared using liquid crystalline template [5]); (b) initial magnetisation of Co structured film.

structure. As can be seen from Fig. 2a the coercivity increases with decreasing pore size for large void diameters. Note that $B_{\mathrm{c}}=2.8 \mathrm{mT}$ for a similarly deposited plain Co film. In the patterned Co sample prepared using 50-nm diameter spheres the coercive field increases to $117 \mathrm{mT}$. After a maximum $B_{\mathrm{c}}$ decreases for films with even smaller pores prepared by a different template method [5]. Our initial results for the coercivity of patterned Co films are consistent with previously observed curves of the dependence of the coercive field vs. magnetic particle size for fine particle magnetic materials $[6,7]$.

The more-usual fine-particle magnetic materials correspond to a complimentary configuration. They are normally termed dot structures. In such case a monodomain magnetic state with coherent rotation of magnetic moments is realised for small grain sizes. In dot structures the peak is normally related to the transition from purely coherent rotation to curling behaviour of magnetic moments [8,9]. For our samples with anti-dot structures (or magnetic networks), the situation is different and a monodomain state is not favoured. In this case the magnetic behaviour is affected by the interaction of domain walls with the voids $[9,10]$. This interaction is sensitive to the relation between magnetic length-scales and pore size. In the case of domain wall pinning, the coercivity reaches an optimum for a pore size close to the domain wall width $w_{\mathrm{B}}$ [9]. For Co the known value $w_{\mathrm{B}}=16 \mathrm{~nm}$ [9] agrees with the radius of spherical pore $R$ corresponding to a maxumum of $B_{\mathrm{c}}$ (Fig. 2a). Our results for different magnetic materials [11] further support the importance of domain wall pinning.

Another important mechanism leading to an increase of $B_{\mathrm{c}}$ originates from a nucleation-controlled barrier. In this case, the formation of a reversed domain is inhibited by high nucleation fields. According to Ref. [9] this mechanism leads to a very steep initial magnetisation branch in contrast to a slow dependence for the case of domain wall pinning. As demonstrated by the results in Fig. $2 b$ the observed initial magnetization is in agreement with a process, for which the domain wall pinning is dominant.

In summary, we have presented results of electrochemical deposition of magnetic metals and alloys in the interstitial spaces of self-assembled templates. The resulting magnetic nanostructures have $3 \mathrm{D}$ achitectures on length scales $50 \mathrm{~nm}-1 \mu \mathrm{m}$. We find large changes in the magnetic parameters resulting from nano-structuring. The template growth technique offers the potential of a low-cost preparation method for sub-micronpatterned magnetic media.

This work has been supported by the EPSRC.

\section{References}

[1] P.N. Bartlett, et al., Chem. Comm. 17 (2000) 1671.

[2] M.A. Ghanem, et al., Chem. Mater 14 (2002) 2199.

[3] M.A. Ghanem, P.N. Bartlett, P.A.J. de Groot, A.A. Zhukov, in preparation.

[4] A.A. Zhukov, et al., Z. fur Phys. B 78 (1990) 195.

[5] P.N. Bartlett, et al., J. Electrochem. Soc. 148 (2001) C119.

[6] F.E. Luborsky, J. Appl. Phys. 32 (1961) 171S.

[7] G. Herzer, IEEE Trans. Magn. 26 (1990) 3327.

[8] D. Craik, Magnetism: Principles and applications, Willey, New York, 1995.

[9] R. Skomski, J.M.D. Coey, Permanent Magnetism, Institute of Physics Publishing, UK, 1999.

[10] J.A. Barnard, et al., Appl. Phys. Lett. 69 (1996) 2758.

[11] A.A. Zhukov, M.A. Ghanem, A.V. Goncharov, H. Fangohr, P.A.J. de Groot, P.N. Bartlett, in preparation. 